\title{
Cost-Efficacy Analysis of Peginterferon alfa-2b plus Ribavirin Compared With Peginterferon alfa-2a plus Ribavirin for the Treatment of Chronic Hepatitis C
}

\author{
DANIEL C. MALONE, PhD; TRAM T. TRAN, MD; and F. FRED POORDAD, MD
}

\begin{abstract}
OBJECTIVE: Combination therapy with pegylated interferon (Peg) and ribavirin (RBV) is the standard of care for the treatment of chronic hepatitis $\mathrm{C}$ virus (HCV) infection. This analysis compares the cost efficacy of treatment with pegylated interferon alfa-2b plus ribavirin (Peg-2b plus RBV) with pegylated interferon alfa-2a plus ribavirin (Peg-2a plus RBV) in hypothetical cohorts of 100 chronic HCV patients comprised $75 \%$ of genotype 1 .
\end{abstract}

METHODS: A decision analysis model was constructed from the viewpoint of a managed care organization to compare Peg-2b plus RBV (1.5 mcg per kilogram per week plus RBV $800 \mathrm{mg}$ per day) and Peg-2a plus RBV (180 mcg per week plus RBV $1,000-1,200 \mathrm{mg}$ per day) pursuant to the label dosing approved by the U.S. Food and Drug Administration. The model also included the so-called weight-based dosing regimen with Peg-2b plus RBV (1.5 mcg per kilogram per week plus RBV $10.6 \mathrm{mg} / \mathrm{kg}$ per day). Patient weight was assumed to be $80 \mathrm{~kg}$. For purposes of this analysis, early virologic response (EVR), defined as viral negative or 2-log drop in viral load, was assessed at 12 weeks for only genotype 1 patients, and nonresponders were assumed to discontinue therapy. The positive predictive value (PPV) was calculated for each treatment group for genotype 1 patients, which is determined from the values for EVR and sustained viral response (SVR). Genotype 2 and genotype 3 patients were assumed to be treated for 24 weeks. Treatment duration and efficacy data were obtained from the published literature. Product pricing was based on average wholesale price, October 2004, and sensitivity analysis was performed using prices from the Federal Supply Schedule. Economic outcomes were determined from hypothetical 100 -patient cohorts assumed to be comprised $75 \%$ of genotype $1 \mathrm{HCV}$.

RESULTS: Taking into account both EVR and SVR, the PPV for genotype 1 patients was 0.63 and 0.57 for Peg-2b plus RBV and Peg-2a plus RBV, respectively. The proportion of treated patients achieving SVR would be nearly identical, (53.6\%) and (53.8\%) for Peg-2a plus RBV and Peg-2b plus flat RBV, respectively. For Peg$2 \mathrm{~b}$ plus weight-based RBV, the proportion of patients achieving SVR was higher (61.4\%). Consequently, this leads to fewer overall treatment weeks for the Peg$2 \mathrm{~b}$ plus RBV cohorts. Therefore, the cost per successful treatment (defined as SVR) was $19.4 \%$ less $(\$ 37,638)$ for Peg-2b plus flat dosing of RBV as compared with Peg-2a plus RBV $(\$ 46,717)$. When Peg-2b plus RBV was dosed $1.5 \mathrm{mcg}$ per kilogram per week plus RBV $10.6 \mathrm{mg} / \mathrm{kg} / \mathrm{day}$, then the cost per SVR was $\$ 39,045$. The cost for the 100-patient cohort was $\$ 2,024,846$ for Peg-2b plus RBV, $\$ 2,397$, 529 for Peg-2b plus weight-based RBV, and \$2,505,317 for Peg-2a plus RBV. This difference is due to a lower PPV in the Peg-2a plus RBV groups and hence more patients treated in spite of a low probability of achieving SVR.

CONCLUSION: The results of this cost-efficacy analysis suggest that treating HCV genotype 1 patients with Peg-2b plus RBV may result in savings to a health care system because fewer of these patients are treated beyond 12 weeks when achieving sustained viral clearance is unlikely.

KEYWORDS: Hepatitis C, Interferon, Peginterferon alfa-2a, Peginterferon alfa-2b, Cost-effectiveness

J Manag Care Pharm. 2005;11(8):687-94
$\mathrm{H}$ epatitis C infection affects 4 million people in the United States and is a common cause of chronic liver disease and its sequelae, including cirrhosis and hepatocellular carcinoma. ${ }^{1}$ The hepatitis C virus (HCV) replicates rapidly, producing $10^{10}$ to $10^{12}$ viral particles a day. ${ }^{2}$ Eradication of the virus requires prolonged treatment with antiviral agents to eliminate the virus in the serum (phase 1 decay) and hepatocytes (phase 2 decay). Response to therapy is measured by a sustained virologic response (SVR), which is defined as the undetectable viral levels 6 months after completion of therapy (Table 1).3,4

Successful treatment of hepatitis $C$ is a function of virus genotype, with patients infected with genotype 1 virus having a much lower probability of SVR than those patients with nongenotype 1 virus. Therapeutic options have evolved from the initial 3-times weekly interferon monotherapy to the current optimal therapy consisting of pegylated (polyethylene glycol molecule) interferon (Peg) alfa in combination with ribavirin (RBV). Pegylated interferons have different pharmacokinetic properties than nonpegylated interferon, including much longer half-life. Thus, pegylated interferons can be dosed once weekly. In 2 large randomized controlled trials of pegylated interferon and RBV versus standard interferon and RBV, the SVR rates were $42 \%$ to $46 \%$ in genotype 1 patients and $76 \%$ to $82 \%$ in genotypes 2 or 3.5,6 Analysis of the data from these 2 pivotal trials determined that once therapy was started, early reduction of viral levels, or early virologic response (EVR) at week 12 of treatment, could predict which genotype 1 patients would be unlikely to achieve SVR., ${ }^{4,7}$ There are numerous advantages to predicting which patients are likely to benefit from treatment

\section{Authors}

DANIEL C. MALONE, PhD, is an associate professor, Colleges of Pharmacy and Public Health, University of Arizona, Tucson; TRAM T. TRAN, MD, is medical director of liver transplantation and F. FRED POORDAD, MD, is chief of hepatology and liver transplantation, Comprehensive Transplant Center, Cedars-Sinai Medical Center, Los Angeles, and both are also assistant professors of medicine, David Geffen School of Medicine, University of California, Los Angeles.

AUTHOR CORRESPONDENCE: Daniel C. Malone, RPh, PhD, Associate Professor, University of Arizona College of Pharmacy, PO Box 210207, Tucson, AZ 85721. Tel: (520) 626-3532; Fax: (520) 626-7355;

E-mail:malone@pharmacy.arizona.edu

Copyright $\odot$ 2005, Academy of Managed Care Pharmacy. All rights reserved. 


\section{TABLE 1 Definitions}

Early virologic response (EVR): a 2 log or greater reduction in hepatitis C RNA levels 12 weeks after the initiation of antiviral therapy.

Sustained virologic response (SVR): the absence of detectible hepatitis C RNA at least 20 weeks after completion of therapy. greater decrease in hepatitis C RNA levels at 12 weeks (early virologic response [EVR]) and also had a sustained virologic response (SVR) after completing therapy Positive predictive value $=$ true positive divided by true positive + false positive. For example: Among a total of 321 patients treated with Peg-2b, 229 had EVR at 12 weeks, with 145 resulting in SVR (true positives) and 84 being false positives. The positive predictive value can be calculated as: $145 /(145+84)=63 \%$.

Negative predictive value (NPV): the proportion of patients who did not have a $2 \log$ or greater decrease in hepatitis C RNA levels at 12 weeks (EVR) and did not achieve an SVR after completing therapy. Negative predictive value $=$ true negative divided by true negative + false negative. In the above example, 92 (321-229) did not have an EVR at 12 weeks. No patients obtained an SVR if they did not have an EVR. The negative predictive value can be calculated as $92 /(92+0)=100 \%$.
Positive predictive value (PPV): the proportion of subjects who had 2 log or

therapy with peginterferon alfa-2b (Peg-2b) (PEG-Intron) plus RBV (Rebetol) and reported that when compared with baseline viral levels, a $2 \log (10)$ (a 100-fold reduction) or greater drop in viral load at 12 weeks of therapy yielded a $72 \%$ positive predictive value (PPV) and a 100\% negative predictive value (NPV) (see Table 1 for definitions of PPV and NPV). ${ }^{4}$ Similar analysis was performed on the peg-interferon alfa-2a (Peg-2a) (Pegasys) plus RBV (Copegus), with a finding of a 65\% PPV and an $97 \%$ NPV. $^{7}$ Therefore, if a 100-fold drop from baseline viral load was not achieved by week 12 of therapy, continuing treatment would be of no benefit because there would be little likelihood of response, and cost as well as side effects could be avoided. Differences in the predictability of viral clearance between Peg-2b and RBV and Peg-2a and RBV may lead to cost differences in treatment because a lower PPV will result in more weeks on treatment when the likelihood of success is low. In other words, a higher initial response (EVR) with a similar final outcome (SVR) means more individuals will be treated for the entire length of therapy even though they will not respond to treatment. Therefore, the purpose of this study was to evaluate the cost efficacy of Peg-2a and Peg-2b when combined with RBV for the treatment of hepatitis $C$ using current practice management algorithms.

\section{Methods}

A decision analytic model was created to compare the cost efficacy of Peg-2a plus RBV (Pegasys + Copegus) and Peg-2b plus RBV (PEG-Intron + Rebetol). The perspective of the analysis was that of a health system (i.e., a managed care organization). The model was constructed to evaluate the costs and outcomes in a cohort of patients with hepatitis C (Figure 1). Three treatment options were evaluated: Peg-2a plus RBV, Peg-2b plus flat dosing of RBV, and Peg- $2 \mathrm{~b}$ plus weight-based dosing of RBV.

The financial time horizon for this particular model was 1 year because of the expected length of treatment for genotype 1. However, response to therapy is typically assessed at approxibeyond 12 weeks, including reduction of treatment side effects, inconvenience, and cost from discontinuation of drug therapy at week 12 .

Davis et al., in a subgroup analysis clinical trial reported by Manns et al., ${ }^{5}$ evaluated viral levels at 4, 12, and 24 weeks of mately 6 months after the last dose of medication. Previously published economic evaluations of hepatitis $C$ therapy have been constructed using lifetime models and have not focused on the short-term costs and outcomes, which are particularly relevant to managed care organizations. Because the reported 
adverse event profiles of these agents are similar, the costs associated with adverse events were not included in the model. The inclusion of treatment of side effects and related costs would increase the complexity of the model but would cancel across the therapies. We also did not include other costs of monitoring that would be similar for the 3 treatment regimens.

\section{Efficacy Data}

The efficacy data came from separate clinical trials involving Peg-2a plus RBV and Peg-2b plus RBV versus standard interferon combination therapy., 50 th trials were multicenter, multinational registration trials using similar study subjects in terms of patient characteristics. For Peg-2a, Fried et al. evaluated $180 \mathrm{mcg}$ of peginterferon alfa-2a given weekly via subcutaneous injection plus daily RBV or placebo for 48 weeks. ${ }^{6}$ The RBV was dosed at 1,000 mg orally per day for those subjects $75 \mathrm{~kg}$ or less and 1,200 mg per day for those subjects weighing more than $75 \mathrm{~kg}$. The comparison arm for this study was interferon 3 million units 3 times weekly plus RBV. Even though 3 groups were evaluated in this trial, the economic model was based upon the data for the Peg-2a plus RBV, the therapy arm of interest. The percentage of patients who discontinued therapy in the study was 22\% for patients receiving Peg-2a plus RBV and 32\% for both groups of interferon plus RBV and Peg-2a plus placebo, depending upon the therapy received. Because all efficacy analyses were based upon intent-to-treat after receiving at least 1 dose, the rate of discontinuation was accounted for in the efficacy analysis.

For Peg-2b, the clinical trial reported by Manns et al. had 3 arms, involving 2 different doses of peginterferon. ${ }^{5}$ One group was treated with peginterferon alfa-2b $1.5 \mathrm{mcg}$ per $\mathrm{kg}$ given weekly subcutaneously plus $800 \mathrm{mg}$ of RBV daily for 48 weeks. The second group was treated with peginterferon alfa- $2 \mathrm{~b}$ given at a dose of $1.5 \mathrm{mcg}$ per $\mathrm{kg}$ weekly for the first 4 weeks of the study, followed by $0.5 \mathrm{mcg}$ per $\mathrm{kg}$ for 44 weeks plus 1,000 to 1,200 mg per day of RBV orally. The third study group received interferon alfa-2b (Intron A) 3 million units subcutaneously 3 times per week plus 1,000 to 1,200 mg of RBV given daily. For the second and third arms, dosing of RBV was based upon subject weight, where those weighing less than $75 \mathrm{~kg}$ received $1,000 \mathrm{mg}$ and those $75 \mathrm{~kg}$ or greater received 1,200 $\mathrm{mg}$. In the United States, Peg-2b was approved based upon dosing of 1.5 mcg per kg for 48 weeks. ${ }^{8}$

A subgroup analysis was performed by Davis et al. on the patients participating in the Peg-2b study (Manns et al.) who received doses of RBV that were at least $10.6 \mathrm{mg}$ per $\mathrm{kg}$ per day, the so-called weight-based dosing regimen. ${ }^{4}$ This analysis found that the higher dose of RBV contributed to a higher response rate. This finding resulted in the weight-based dosing regimen becoming the standard of care outside the United States. The reported discontinuation rate in this study ranged from $13 \%$ to
14\% depending upon the treatment arm. ${ }^{5}$ Efficacy assessment in this clinical trial was based upon intention to treat after the first dose received.

In the clinical trials reported by Manns et al. and Fried et al., SVR was the primary end point, defined as no detectable HCV RNA in the serum 24 weeks after cessation of drug therapy. In addition, both studies evaluated early viral response (EVR).

Davis et al., in the subgroup analysis of the Manns et al. trial, defined various thresholds as EVR and found that either a 1 or 2 log decline in HCV RNA at 12 weeks showed the highest sensitivity and also the highest NPV (excluding those persons who did not respond to treatment). ${ }^{4}$ For the present cost-efficacy analysis, we defined EVR as a 2-log decrease from baseline in HCV RNA levels after 12 weeks of treatment. Consequently, the PPV of EVR affects the overall cost of treatment when treatment is adjusted according to the EVR results. PPV is defined as the likelihood of achieving SVR among those persons who do achieve a rapid virologic response. Evaluating the EVR is important for those patients with the genotype 1 virus because patients destined to fail therapy can be discontinued early. The 12-week rule in genotypes 2 and 3 is less useful because of the shorter treatment course and higher response rates. In fact, it is being modified to a 4-week assessment, or rapid virological response (RVR). Thus, this model specifically accounts for the ratio of genotype 1 to genotype 2 or genotype 3 in both studies and the PPV in response to treatment for persons with genotype 1.

\section{Model Specification}

The primary model examined 2 hypothetical cohorts of 100 $\mathrm{HCV}$ subjects receiving either Peg-2a or Peg-2b. One cohort received 180 mcg weekly of Peg-2a plus RBV 1,000 to 1,200 mg daily, the second received $1.5 \mathrm{mcg}$ weekly of Peg-2b plus 800 mg of RBV daily (so-called flat dosing). We also extended the model to include a third cohort that reflects dosing not approved for the product label in the United States in which patients receive $1.5 \mathrm{mcg}$ weekly of Peg-2b plus $10.6 \mathrm{mg} / \mathrm{kg}$ of RBV daily (the so-called weight-based dose). For all 3 cohorts, a patient weight of $80 \mathrm{~kg}$ was assumed. The proportion of patients with genotype 1 was assumed to be $75 \%$. ${ }^{1}$ This value is higher than those reported in clinical trials with peginterferons, but it was believed to be more representative of the U.S. population because the clinical trials were conducted internationally and genotype 1 has a high prevalence in the U.S. as compared with some other parts of the world where the clinical trials were conducted. For example, prevalence of genotype I is $48.7 \%$ in Belgium $^{9}$ and $57.9 \%$ in France. ${ }^{10}$ Prevalence of type 1 genotype HCV is similar in Japan and the United States, but lower in Brazil, Vietnam, and Indonesia. ${ }^{11}$

For those subjects with non-genotype 1, it was assumed that treatment lasted 24 weeks. For genotype 1 patients, it was assumed that viral response was assessed at 12 weeks. Those patients who had a decrease of 2 logs or more in viral load were 


\section{TABLE 2 Patient Demographics From Clinical Trials}

\begin{tabular}{|c|c|c|c|}
\hline Characteristics & $\begin{array}{c}\text { Peginterferon } \\
\text { alfa- } 2 \mathrm{a} \\
180 \mathrm{mcg}+ \\
1,000 \text { to } 1,200 \mathrm{mg} \\
\text { Ribavirin }^{6} \\
\mathrm{~N}=453\end{array}$ & $\begin{array}{c}\text { Peginterferon } \\
\text { alfa- } 2 \mathrm{~b} \\
1.5 \mathrm{mcg} / \mathrm{kg}+ \\
1,000 \text { to } 1,200 \mathrm{mg} \\
\text { Ribavirin }^{5} \\
\mathrm{~N}=511\end{array}$ & $\begin{array}{c}\text { Peginterferon } \\
\text { alfa-2b } \\
1.5 \mathrm{mcg} / \mathrm{kg}+ \\
10.6 \mathrm{mg} / \mathrm{kg} \\
\text { Ribavirin } \\
\mathrm{N}=188\end{array}$ \\
\hline $\begin{array}{l}\text { Sex: male/female } \\
(\% \text { male) }\end{array}$ & $324 / 129(72)$ & $321 / 190(63)$ & NR \\
\hline Age (years) & $42.8 \pm 10.1$ & $43.9 \pm 8.0$ & $42.8 \pm 8.7$ \\
\hline Weight (kg) & $79.8 \pm 17.5$ & $82.4 \pm 18.0$ & $64.5 \pm 7.5$ \\
\hline $\begin{array}{l}\text { Mean HCV RNA in } \\
\text { serum (copies/ml } \\
\left.\times 10^{-6}\right)\end{array}$ & 6.0 & $6.4 \pm 0.6$ & $6.3 \pm 0.6$ \\
\hline Genotype 1: n (\%) & 298 (65) & 348 (68) & $122(65)$ \\
\hline
\end{tabular}

Davis et al. ${ }^{4}$ is a subgroup analysis of the trial reported by Manns et al. ${ }^{5}$ $H C V=$ hepatitis $C$ virus; $N R=$ not reported.

\section{Costs}

The model took into account only the costs of peginterferon and RBV since all other treatment resources would be similar. Medication costs were based upon average wholesale price (AWP) as listed by Medispan (effective October 2004). The price of Peg-2a was $\$ 401.04$ per dose. For Peg-2b, the $120 \mathrm{mcg}$ dose was $\$ 406.94$. The $120 \mathrm{mcg}$ dose of Peg-2b was selected because this strength is recommended for patients weighing between 61 to $85 \mathrm{~kg}$ when receiving combination therapy with $\mathrm{RBV}^{8}$ The price of RBV has been affected by the patent expiration, but at the time of this analysis (October 2004), the prices for generic formulations were higher than for the brand-name (Copegus). Therefore, we used the brand-name price for RBV, which was $\$ 6.64$ per $200 \mathrm{mg}$ capsule. Managed care organizations typically reimburse pharmacies or purchase pharmaceuticals at substantially less than AWP. To account for this, we conducted a sensitivity analysis using AWP minus $17 \%$. Under this situation, the costs were $\$ 332.86$ for Peg-2a (83\% of $\$ 401.04$ ), $\$ 337.76$ for Peg-2b (83\% of $\$ 406.94$ ), and $\$ 5.50$ for RBV (83\% of $\$ 6.63$ ).

Another sensitivity analysis was conducted using the Federal Supply Schedule prices (FSS) as obtained from the Department of Veterans Affairs Pharmacy Benefits Management Group. ${ }^{12}$ Product pricing under the FSS was $\$ 143.30$ for Peg-2a, $\$ 126.53$ for Peg-2b, and $\$ 1.00$ per dose for RBV. The FSS, with its best price provision, represents the lowest prices that the government or any managed care organization would pay for these products (excluding donated products or samples).

Sensitivity analyses were conducted to determine the impact of variable uncertainty on the models. One-way sensitivity analyses were performed. Threshold analysis was done for the key parameters of PPV and peginterferon costs. Threshold analysis is a 1-way sensitivity analysis where a parameter is varied until a break-even point is reached, ignoring the plausible range for that particular variable.

\section{Results}

Patient characteristics in the 3 treatment groups in the 2 clinical trials were similar except for patient weight, which was considerably lower in the weight-based RBV and Peg-2b group compared with the others (Table 2). Patients in this weightbased RBV group were those who received at least $10.6 \mathrm{mg} / \mathrm{kg}$ of RBV, and, since the dosing regimen was $800 \mathrm{mg}$ for all patients, they were, by definition, lighter in average body weight. Across the 3 groups, the percentage of patients that were genotype 1 ranged from $65 \%$ to $68 \%$.

Table 3 shows the SVR for all subjects and for genotype 1 subjects across the 3 groups. SVR among genotype 1 subjects was higher for Peg-2a (46\%) than Peg-2b flat dose (42\%), but Peg-2b with weight-based dose RBV had the highest SVR (48\%). However, these SVRs were not significantly different from each other based upon the 95\% confidence intervals.

Peg-2a had the highest EVR, with $81 \%$ of genotype 1 subjects 
showing a response to therapy after 12 weeks (Table 3). For Peg-2b, the EVR was $71 \%$ for flat RBV dosing and $74 \%$ for weight-based RBV dosing. The PPV for Peg-2a for genotype 1 was $57 \%,{ }^{7}$ as compared with $63 \%$ and $65 \%$ for Peg-2b flat and weight-based dosing, respectively (Table 3). ${ }^{4}$ Thus, even though Peg-2a has a larger percentage of genotype 1 patients that have an initial viral decline (at week 12), the relapse rate is higher, and, hence, the overall SVR for Peg-2a (40\% to 52\%) is similar to Peg-2b (37\% to $47 \%$ for flat dosing of RBV and 39\% to $57 \%$ for weight-based dosing of RBV) (see Table 3). It is important to note that, in the clinical trials, the EVR stopping rules were not in place. Thus, all genotype 1 patients were assigned to receive 48 weeks of treatment. The important variable that influences the results of this study is that because of the higher EVR for Peg-2a, more patients are treated who are unlikely to benefit, which increased the cost for the Peg-2a cohort.

Estimates of the direct drug cost of each treatment regimen and genotype are shown in Table 4. For all 3 treatment regimens, the drugs costs of treatments are fairly similar, with a full year (48 weeks) of treatment for each genotype 1 patient from $\$ 28,444$ to $\$ 32,999$ and from $\$ 14,222$ to $\$ 16,450$ for each genotype 2 or 3 patient for 24 weeks of drug therapy.

The results from the economic analysis are shown in Table 5. The costs of drug therapy for each 100-patient cohort ranges from $\$ 2.02$ million for Peg-2b + fixed dose RBV to $\$ 2.51$ million for Peg-2a + RBV. The difference in cost was a result of total number of weeks on therapy: 3,687 weeks of therapy for Peg-2a compared with 3,417 for Peg-2b flat or 3,498 for weight-based regimens. The difference in cost for a cohort of 100 patients between Peg-2b flat dosing and Peg- $2 \mathrm{a}$ is $\$ 480,000$. This $\$ 480,000$ is equivalent to an additional 17 genotype 1 patients being treated for 48 weeks using Peg-2b + fixed-dose RBV.

The proportion of patients who achieve SVR was similar among Peg-2a (53.63\%) and Peg-2b flat dosing (53.80\%), and higher for Peg-2b weight-based dosing (61.41\%). There was a difference of almost 8 patients between Peg- $2 \mathrm{~b}$ plus flat RBV and weight-based RBV treatments. The cost to achieve a successfully treated patient, defined as having an SVR, was lowest with Peg-2b flat dosing at a cost of $\$ 37,638$. Peg-2b weightbased RBV dosing had a cost/SVR of $\$ 39,045$, and Peg-2a had a cost/SVR of $\$ 46,717$. The incremental cost-effectiveness of using Peg-2b weight-based dosing over Peg-2b flat dosing was $\$ 48,989$ for each additional patient obtaining an SVR. Peg-2a was less cost effective than Peg-2b flat and weight-based treatments.

\section{Sensitivity Analysis}

One-way sensitivity analyses were conducted to evaluate the input of key parameters on the model. One of the key parameters for the model was the PPV for genotype 1 . If the PPV for Peg-2a is increased from 0.57 to 0.63 , then the number of successfully treated patients for Peg-2a was 57.27, an increase of 3.64. The resulting cost per successfully treated patient was $\$ 43,744$ for

\begin{tabular}{|c|c|c|c|}
\hline Regimen & $\begin{array}{c}\text { Genotypes } 2 \& 3 \\
24 \text { weeks }(\$)\end{array}$ & $\begin{array}{c}\text { Genotype } 1 \\
12 \text { weeks (\$) }\end{array}$ & $\begin{array}{l}\text { Genotype } 1 \\
48 \text { weeks (\$) }\end{array}$ \\
\hline $\begin{array}{l}\text { Peg-2a + RBV } \\
1,000 \text { to } 1,200 \text { mg daily }\end{array}$ & 16,308 & 8,154 & 32,616 \\
\hline $\begin{array}{l}\text { Peg- } 2 b+\text { RBV } \\
800 \text { mg daily }\end{array}$ & 14,222 & 7,111 & 28,444 \\
\hline $\begin{array}{l}\text { Peg-2b + RBV } \\
\geq 10.6 \mathrm{mg} / \mathrm{kg} \text { daily }\end{array}$ & 16,450 & 8,225 & 32,899 \\
\hline
\end{tabular}

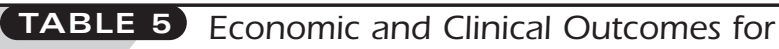
100-Patient HCV Cohorts

\begin{tabular}{|c|c|c|c|c|}
\hline Regimen & $\begin{array}{l}\% \text { Patients } \\
\text { with SVR* }\end{array}$ & $\begin{array}{c}\text { Cost/SVR } \\
(\$)\end{array}$ & $\begin{array}{l}\text { Weeks of } \\
\text { Therapy }\end{array}$ & $\begin{array}{l}\text { Total Cohort } \\
\text { Cost }(\$)\end{array}$ \\
\hline $\begin{array}{l}\text { Peg-2a + RBV } \\
1,000 \text { to } 1,200 \mathrm{mg} \text { daily }\end{array}$ & 53.63 & 46,717 & 3,687 & $2,505,317$ \\
\hline $\begin{array}{l}\text { Peg-2b+ RBV } \\
800 \text { mg daily }\end{array}$ & 53.80 & 37,638 & 3,417 & $2,024,846$ \\
\hline $\begin{array}{l}\text { Peg-2b + RBV } \\
\geq 10.6 \mathrm{mg} / \mathrm{kg} \text { daily }\end{array}$ & 61.41 & 39,045 & 3,498 & $2,397,529$ \\
\hline
\end{tabular}

Peg-2a, still more than for Peg-2b flat or weight-based dosing. The incremental cost-efficacy ratio was $\$ 138,265$ per successful treatment. The threshold value for Peg-2a PPV was 0.78, which was well beyond the $95 \%$ confidence interval of 0.51 to 0.63 . Another analysis evaluated the PPV of Peg-2b flat dosing. When the PPV is only 0.43 , then the cost per successful outcome for Peg-2a and Peg-2b flat dosing is nearly identical. Again, this is well beyond the 95\% confidence interval for the PPV for Peg- $2 \mathrm{~b}$ flat dosing.

Other sensitivity analyses were conducted examining the cost of the various treatments. If product pricing was at AWP minus $17 \%$, then the cost per successfully treated patient for the 3 treatments was $\$ 31,240, \$ 32,407$, and $\$ 38,775$ for Peg- $2 b$ flat dosing, Peg-2b weight-based dosing, and Peg-2a, respectively. In the incremental analysis, use of Peg-2a was less cost effective than Peg-2b and weight-based dosing of RBV. When FSS prices are applied to all therapies, including the price of RBV, the lowest cost per successfully treated patient was Peg-2b weight-based dosing ( $\$ 12,630$ per successfully treated patient). Peg-2a was only $\$ 110$ more, at $\$ 12,740$ per SVR, while Peg-2b flat dosing was $\$ 13,193$ per SVR. Using FSS prices, Peg-2b plus flat RBV dosing had the lowest cohort cost $(\$ 528,029$ versus $\$ 683,201$ 
for Peg-2a). Under FSS pricing, Peg-2a was again more costly and less effective than Peg-2b flat- and weight-based dosing regiments.

A threshold analysis was conducted varying the AWP price of Peg-2a. When it was reduced to $\$ 269$ (a reduction of $\$ 138$ [34\%]) per $180 \mathrm{mcg}$, then the cost per successfully treated patient was identical for Peg-2a and Peg-2b flat dosing. Varying patient weight to the lower and upper bounds of the 95\% confidence interval as well as varying the proportion of genotype 1 patients to $50 \%$ did not affect the rank order of the results in terms of cost per successfully treated patient.

\section{Discussion}

The results from this cost-efficacy study suggest that treatment with Peg-2b may be less costly than with Peg-2a for a cohort of subjects with $\mathrm{HCV}$ who are eligible for treatment. Although both Peg-2a and Peg-2b have demonstrated similar SVR overall and by genotype, for genotype 1 , there is a significant difference in EVR rates between Peg-2a and Peg-2b flat dosing. In this analysis, the higher EVR for Peg-2a among genotype 1 patients leads to more treatment but with no additional benefit over those treated with Peg-2b flat- or weight-based dosing. Consequently, the cost per successful treatment is lower with Peg-2b combination therapy as compared with Peg-2a combination therapy. The results of the sensitivity analyses indicated that efficacy rates or costs would have to change substantially to affect the rank order of the products.

Pegylated interferon plus RBV is now the standard of care for the treatment of chronic hepatitis C. Durable viral eradication is possible with effective therapy in more than $50 \%$ of patients; however, cost and side effects may limit the number of patients successfully treated. Viral kinetic studies have shown that once interferon therapy is initiated, an early rapid initial decline in viral levels, termed phase 1 decay, is noted, but this decline does not correlate well to eventual viral clearance. It is the subsequent phase 2 decay that takes place over the next several weeks that reflects the death rate of infected hepatocytes and does correlate with sustained virologic response., ${ }^{3,13}$ This understanding of the viral kinetic profile led to analysis of the viral response at 12 weeks into therapy in both pivotal pegylated interferon plus RBV trials. From these data, PPVs and NPVs have been determined; Peg-2b has a higher PPV than does Peg-2a, with a similar NPV. The economic differences are a function of this difference in PPV.

Quantitative HCV RNA testing has become widely available and has made the clinical use of viral load feasible. Although there are some limitations to these tests, including different assays and limited dynamic linear ranges of the tests, there are now important clinical applications to testing. Prior to initiating therapy, a baseline viral load should be obtained. In the genotype 1 patient, after 12 weeks of therapy another viral load is obtained and compared with the patient's baseline viral load.
If an EVR, defined as at least a $2 \log 10$ drop from baseline viral load, is achieved, then the patient should be continued on therapy because they have a good chance of SVR at the end of followup. If, however, an EVR is not achieved at 12 weeks, an SVR is highly unlikely, and the patient should not be put through the cost and potential side effects of the full 48 weeks of treatment.

In some clinical scenarios, such as the patient with advanced fibrosis or extrahepatic hepatitis $\mathrm{C}$ disease, patients may be continued on therapy with modified doses of interferon, not with the goal of viral eradication but with the goal of viral suppression. Current ongoing prospective studies will assess the utility of this practice.

A recent article by Mangia et al. reported the results of testing for HCV RNA levels at week 4 for genotype 2 and 3 patients treated with Peg-2b plus RBV. ${ }^{14}$ The study had 2 primary groups: 24 weeks of treatment or HCV RNA testing at 4 weeks. For the group with 4-week HCV RNA assessments, treatment was continued for either 12 or 24 weeks depending upon the result of the test (undetectable levels resulted in 12 weeks of additional therapy; whereas HCV positive patients continued treatment for 24 weeks). The overall response rate for early response was $62 \%$ versus $64 \%$ for usual treatment for 24 weeks (no HCV RNA testing).

The use of the EVR applies to all genotypes; however, since recent data have shown an excellent response to only 24 weeks of therapy in genotypes 2 and 3, the use of the 12-week EVR is not of significant cost benefit. It is mainly in the more difficultto-treat genotype 1 patients who require 48 weeks of therapy that an early-stopping rule is most useful. The 2002 National Institutes of Health Consensus Development Conference Statement on Hepatitis C has recommended the use of EVR stopping rules, and prospective validation of these data is forthcoming in ongoing studies. ${ }^{15}$ Consequently, the economic rationale for using one peginterferon over another is based not solely on EVR but, rather, the likelihood that patients will respond to the full course of therapy. Therefore, even though Peg-2a has an initial higher EVR than Peg-2b, there is no difference in SVR achieved when compared with Peg-2b, making the latter a more cost-effective therapy.

In our analysis, using the published data from the Manns et al. ${ }^{5}$ and Fried et al. ${ }^{6}$ clinical trials, the calculated PPVs and EVR data led to a difference in our theoretical cohort of number of patients who continued on therapy after an EVR was achieved but would not ultimately achieve an SVR. Therefore, in this cost analysis, it was more costly to treat a cohort of 100 patients with Peg-2a and RBV because more patients were continued on therapy who did not ultimately achieve viral eradication. The reason for this difference may be related to the higher relapse rates noted in the Peg-2a trial.

Previous economic analyses have examined Peg-2a plus RBV and Peg-2b plus RBV as compared with standard interferon therapy plus RBV. ${ }^{16-20}$ Most of these studies were conducted 
using international cost data, with Sullivan et al. using cost structures from Italy ${ }^{16}$; Buti et al. based upon structures from Spain ${ }^{17}$; and Siebert et al. using a German perspective. ${ }^{18}$ Wong et al. ${ }^{19}$ and another paper by Sullivan et al. ${ }^{20}$ use cost values that were specific to the U.S. market. All analyses found that peginterferon plus RBV was cost effective relative to standard interferon plus RBV. Wong et al. found that Peg-2b was incrementally cost effective compared with interferon alfa-2b, at a cost of $\$ 13,600$ to $\$ 22,800$ per quality-adjusted life-year (QALY). ${ }^{19}$ The U.S.-based analysis by Sullivan et al..$^{20}$ found that the incremental costeffectiveness ratio of Peg-2a plus RBV compared with interferon alfa-2b was $\$ 2,600$ per QALY. The other economic analyses have found similar costs (approximately 10,894/ QALY in Italy ${ }^{16} ; 3,760 /$ QALY in Spain ${ }^{17}$; and 6,600/QALY in Germany ${ }^{18}$ ). By conventional standards, peginterferon is cost effective relative to standard interferon. ${ }^{21}$ Therefore, the decision becomes one of which peginterferon to use.

\section{Limitations}

This analysis was based on publicly available information at the time the study was conducted and data from 2 large clinical trials. One of the clear limitations, therefore, is the differences in study criteria and patient populations, although most patient characteristics were fairly similar (Table 2 ). In the clinical study by Freid et al., study patients were eligible if they met the following criteria: were interferon naive; had at least 2,000 copies of HCV RNA per milliliter of serum, their serum alaminotransferase activity was greater than the upper limit of normal within 6 months of study entry; and they had a liver biopsy result consistent with the diagnosis of chronic hepatitis C. ${ }^{6}$ Patients were excluded from the study if they had any of the following: neutropenia, thrombocytopenia, anemia, HIV infection, decompensated liver disease, serum creatinine level greater than 1.5 times the upper limit of normal, poorly controlled psychiatric disease, alcohol or drug dependence within a year of entering the study, or a substantial coexisting medical condition. The criteria for entry into the Manns et al. study included the following: previously untreated adult patients with a liver-biopsyconfirmed diagnosis of chronic hepatitis C; serum alanine aminotransferase levels above the upper limit of normal; absence of neutropenia, anemia, or thrombocytopenia; and bilirubin, albumin, and creatine within normal limits. ${ }^{5}$ Patients were excluded from the Manns et al. trial if they had any of the following: decompensated cirrhosis, serum alpha-fetoprotein of more than $50 \mu \mathrm{g}$ per liter, HIV infection, previous organ transplantation, other causes of liver disease, hemophilia, poorly controlled diabetes, and autoimmune disorders; if they were unable to use contraception; or had any of the following diseases or conditions: psychiatric disease, seizures, cardiovascular disease, or hemoglobinopathies. In general, these inclusion and exclusion criteria are comparable between the studies.

Second, this analysis excluded consideration of adverse events that may result from treatment. The types of adverse events experienced by patients exposed to interferon and peginterferon are similar, but the actual frequency of specific adverse events may vary. For example, fever was reported in $43 \%$ of subjects receiving Peg- $2 \mathrm{a}^{6}$ and $46 \%$ of subjects receiving Peg-2b. ${ }^{5}$ The incidence of neutropenia was $20 \%$ for Peg- $2 \mathrm{a}^{6}$ and $18 \%$ for Peg- $2 \mathrm{~b} .^{5}$ In addition, criteria for classifying adverse events was not well defined in the publications for either trial. Finally, there have been no published data of large studies ( $>60$ patients per group) comparing Peg- $2 \mathrm{a}$ and Peg- $2 \mathrm{~b}$ in a head-to-head fashion, though a multicenter, randomized study is currently ongoing. Until data are available from these directcomparison studies, economic analyses need to be based upon the existing available evidence to assist in the efficient allocation of resources. ${ }^{22}$

Another issue with decision models is the use of deterministic cost data, and especially the use of AWP. To overcome this limitation, we used a price of AWP minus $17 \%$ and also prices from the FSS to represent the range of possible costs for managed care organizations. These analyses also included changing the cost of RBV. Organizations should carefully evaluate this model in context with the prices actually paid for these agents and other factors that may influence product use.

\section{Conclusion}

This study found that the use of Peg- $2 b$ and RBV may be preferred to Peg-2a because of its lower cost of treatment for a hypothetical cohort of $100 \mathrm{HCV}$ patients. The primary factor in the analysis was the difference in EVR and PPV, which led to fewer genotype 1 patients in the Peg-2b cohort continuing treatment when there was a very low likelihood of eventual sustained virologic response.

\section{DISCLOSURES}

Funding for this research was provided by Schering-Plough Pharmaceuticals, Inc. and was obtained by author Daniel C. Malone. Malone discloses that he is a consultant to Schering Plough; author Tram T. Tran discloses that she has have received grants and honoraria from Schering Plough; F. Fred Poordad discloses that he has have received grants and honoraria from and done research for Schering-Plough.

Malone served as principal author of the study. Study concept and design were contributed by Malone and Tran. Analysis and interpretation of data were contributed by all authors; statistical expertise was contributed by Malone. Drafting of the manuscript was primarily the work of Malone, and its critical revision was the work of all authors. Administrative, technical, and/or material support was provided by Jaime Pew, Schering-Plough.

\section{REFERENCES}

1. Alter MJ, Kruszon-Moran D, Nainan OV, et al. The prevalence of hepatitis C virus infection in the United States, 1988 through 1994. N Engl J Med. 1999; 341:556-62.

2. Davis GL. Hepatitis C virus genotypes and quasispecies. Am J Med. 1999; 107(6B):21S-26S

3. Neumann AU, Lam NP, Dehari $H$, et al. Hepatitis $C$ viral dynamics in vivo and the antiviral efficacy of interferon alfa therapy. Science. 1998:282:103-07. 


\section{Cost-Efficacy Analysis of Peginterferon alfa-2b plus Ribavirin Compared With Peginterferon alfa-2a plus Ribavirin for the Treatment of Chronic Hepatitis C}

4. Davis GL, Wong JB, McHutchison JG, et al. Early virologic response to treatment with peginterferon alfa-2b plus ribavirin in patients with chronic hepatitis C. Hepatology. 2003;38:645-52.

5. Manns MP, McHutchison JG, Gordon SC, et al. Peginterferon alfa-2b plus RBV compared with interferon alfa-2b plus ribavirin for initial treatment of chronic hepatitis C: a randomised trial. Lancet. 2001;358:958-65.

6. Fried MW, Shiffman ML, Reddy KR, et al. Peginterferon alfa-2a plus ribavirin for chronic hepatitis C virus infection. N Engl J Med. 2002;347: 975-82.

7. Ferenci P, Shiffman ML, Fried MW, et al. Early prediction of response to 40 KDA peginterferon alfa-2a (Pegasys) plus ribavirin in patients with chronic hepatitis C. Poster presented at: 2001 American Association for the Study of Liver Diseases; November 11, 2001; Dallas, TX. Poster 716

8. PEG-Intron (Peginterferon alfa-2b) Powder for Injection PI/MedGuide. Kenilworth NJ: Schering Corporation; revised June 2004.

9. Mathei C, Wollants E, Verbeeck J, et al. Molecular epidemiology of hepatitis $\mathrm{C}$ among drug users in Flanders, Belgium: association of genotype with clinical parameters and with sex- and drug-related risk behaviors. Eur J Clin Microbiol Infect Dis. August 19, 2005 [epub ahead of print].

10. Cantaloube JF, Gallian P, Attoui H, et al. Genotype distribution and molecular epidemiology of hepatitis $C$ virus in blood donors from southeast France. J Clin Microbiol. 2005;43:3624-29.

11. Nakano T, Lu L, Liu P, Pybus OG. Viral gene sequence reveal the variable history of hepatitis C virus infection among countries. J Infect Dis. 2004;190: 1098-1108.

12. Pharmacy Benefits Management Strategic Healthcare Group, Department of Veterans Affairs. Available at: http://www.vapbm.org/PBM/menu.asp. Accessed June 24, 2004.

13. Zeuzem S, Herrmann E. Dynamics of hepatitis C virus infection. Ann Hepatol. 2002;1:56-63.
14. Mangia A, Santoro R, Minerva N, et al. Peginterferon alfa-2b and ribavirin for 12 vs. 24 weeks in HCV genotype 2 or 3. N Engl J Med. 2005;352:260917

15. Management of hepatitis C: 2002. NIH Consensus and State-of-theScience Statements 2002;19(3):1-46.

16. Sullivan SD, Jensen DM, Bernstein DE, et al. Cost effectiveness of peginterferon alfa-2a plus ribavirin versus interferon alfa-2b plus ribavirin as initial therapy for treatment-naïve chronic Hepatitis C. Pharmacoeconomics. 2004; 22:257-65

17. Buti M, Medina M, Casado MA, et al. A cost-effectiveness analysis of peginterferon alfa- $2 \mathrm{~b}$ plus ribavirin for the treatment of naïve patients with chronic hepatitis C. Aliment Pharmacol Ther. 2003;17:687-94.

18. Siebert U, Sroczynski G, Rossol S, et al. Cost effectiveness of peginterferon alfa- $2 \mathrm{~b}$ plus ribavirin versus interferon alfa- $2 \mathrm{~b}$ plus ribavirin for initial treatment of chronic hepatitis C. Gut. 2003;52:425-32.

19. Wong JB, Davis GL, McHutchison, et al. for International Hepatitis Interventional Therapy Group. Economic and clinical effects of evaluating rapid viral response to peginterferon alfa-2b plus ribavirin for the initial treatment of chronic hepatitis C. Am J Gastroenterol. 2003;98:2354-62.

20. Sullivan SD, Jensen DM, Bernstein DE, et al. Cost-effectiveness of combination peginterferon alfa- $2 \mathrm{a}$ and ribavirin compared with interferon $\mathrm{alf} a-2 \mathrm{~b}$ and ribavirin in patients with chronic hepatitis C. Am J Gastroenterol. 2004; 99: 1490-96.

21. Siegel JE, Weinstein MC, Torrance GW. Reporting cost-effectiveness studies and results. In: Gold MR, Siegel JE, Russell LB, Weinstein MC, ed. CostEffectiveness in Health and Medicine. New York: Oxford University Press. 1996:276-303.

22. Gabriel S, Drummond M, Maetzel A, Boers M, Coyle D, Welch V, Tugwell P. OMERACT 6 Economics Working Group Report: A proposal for a reference case for economic evaluation in rheumatoid arthritis. J. Rheumatol. 2003;30 886-90 